\title{
Modelo conjunto de calidad de vida laboral y salud mental en el trabajo: Valor explicativo del modelo y de sus escalas
}

\author{
QUALITY WORK-LIFE AND OCCUPATIONAL MENTAL HEALTH \\ INTEGRATED MODEL: MODEL AND MODEL'S SCALES VALIDITY
}

\section{Antonio Duro Martín*}

\section{RESUMEN}

El objetivo del presente estudio es examinar la validez explicativa general del modelo de calidad de vida laboral y salud mental en el trabajo, y de sus escalas de medida. Este modelo incluye cuatro grupos de condiciones estructurales: condiciones de trabajo, condiciones de adaptación, condiciones de ajuste, y condiciones de experiencia subjetiva (bienestar y salud laborales); y un grupo de condiciones moduladoras. Utilizando una muestra de 719 trabajadores, y un diseño transversal, se contrastó la significatividad de la varianza explicada de las escalas de bienestar laboral psicológico y salud mental en el trabajo por parte de las restantes escalas del modelo. Los resultados apoyan que las escalas de medida de las condiciones de trabajo, condiciones de adaptación, condiciones de ajuste, y condiciones moduladoras explican significativamente el bienestar y salud de los trabajadores. Se concluye que el modelo resulta útil para la evaluación primaria de riesgos psicosociales en la empresa.

Palabras clave: Calidad de vida laboral, bienestar laboral, salud mental laboral, síntomas psicológicos debidos al trabajo, stress laboral, burnout, acoso, características del trabajo

\section{AbSTraCt}

This study's propose is to test the validity of worklife and occupational health model's measurement scales. This model includes four groups of structural conditions: work conditions, adaptation conditions, matching work-worker conditions, and subjective experience conditions (occupational well-being and health); and one group of modulating conditions. Using a 719 workers sample, and a cross-sectional design, we tested the significance of the explained variance of the occupational well-being and work-related mental ill-health scales by means of the resting model's scales. Results support that the measurement scales of the work conditions, adaptation conditions, matching work-worker conditions, and modulating conditions explain significantly the workers' occupational well-being and health. It is concluded that the model is useful for a primary psychosocial risks evaluation in companies.

Key words: Quality of work life, employee's well-being, occupational mental health, occupational stress, burnout, mobbing, harassment, job conditions

Licenciado en Psicología, de la Universidad Autónoma de Madrid, Facultad de Psicología, 1984. Psicologo, de la Universidad Autónoma de Madrid, Facultad de Psicología, 1985. Doctor en Psicología, de la Universidad Autónoma de Madrid, Facultad de Psicología, 1998. Actualmente, profesor Asociado del Departamento de Ciencias Sociales y Humanidades de la Universidad Rey Juan Carlos. Madrid España.

Correo Electrónico: antonio.duro@urjc.es

Artículo Tipo 1: de Investigación Cientifica y Tecnológica. Según Clasficación Colciencias.

Fecha de Recepción: 5 de mayo del 2008 


\section{INTRODUCCIÓN}

El modelo conjunto de calidad de vida laboral y salud mental en el trabajo es un modelo de carácter integrado para explicar simultáneamente el bienestar laboral psicológico (BLPS) y la salud mental laboral (SML) de los trabajadores. Sus fundamentos teóricos ya han sido expuestos en otro lugar (Duro, 2005a), y sus resultados descriptivos preliminares coinciden con los resultados obtenidos en otras investigaciones previas sobre este mismo campo, lo que apoya su validez concurrente (Duro, 2005b). Por otra parte, la estructura factorial de sus escalas de medida proporciona apoyo a su validez de constructo (Duro, 2005c; 2007). Sin embargo, hasta la fecha no se ha comprobado expresamente su validez explicativa, esto es: ¿En qué medida explica realmente el modelo el BLPS y la SML de los trabajadores? El presente estudio aborda precisamente este problema, utilizando los datos procedentes de una muestra de más de 700 trabajadores.

\section{Estructura DEL MODELO}

El modelo de calidad de vida laboral y salud mental en el trabajo incluye como condiciones antecedentes del bienestar y salud de los trabajadores sus respectivas (a) condiciones de trabajo -concebidas como input laboral-, (b) sus condiciones de adaptación -que recogen los recursos e interpretaciones del trabajador-, y (c) las condiciones de ajuste trabajo-trabajador resultantes -que reflejan el ajuste o discrepancia logrado entre los valores reales y los valores deseados de las dos condiciones anteriores. Las condiciones de experiencia subjetiva son las condiciones consecuentes finales, y contienen justamente el BLPS y la SML de los trabajadores (ver figura 1). En rigor, el BLPS recoge todas aquellas consecuencias del trabajo sobre el trabajador de carácter no clínico, en tanto que la SML recoge a su vez todas las consecuencias clínicas que sufre el trabajador por causa de su trabajo. Además, este modelo incorpora un conjunto de condiciones moduladoras que actúan de manera específica sobre ciertas condiciones de trabajo y de experiencia subjetiva.

Todas estas condiciones se derivan y tienen su origen en la literatura sobre calidad de vida laboral y salud en el trabajo (Duro, 2005a). Especialmente, el modelo incorpora diversas aportaciones teóricas provenientes del (a) estrés (p.ej., Cooper, 1998; Cooper \& Marshall, 1978; Karasek, 1979; Peiró, 1993; Spielberger, 1999; Spielberger, Vagg \& Wasala, 2003), (b) del burnout (p.ej., Freudenberger, 1974; Lee \& Ashforth, 1996; Maslach, 1982, 1986; Maslach \& Jackson, 1981; Phillips, 1984; Pines \& Aronson, 1988; Shirom, 1989; Shirom, 2003), (c) del acoso moral (mobbing) (Piñuel \& Zabala, 2001),(d) del acoso sexual en el trabajo (Harned et al, 2002), y (e) de otros temas afines a los anteriores - p.ej., matonería (bullying) y hostilidad en el puesto de trabajo (Hodson, Roscigno, \& López, 2006). Al propio tiempo, las condiciones componentes del modelo se conceptúan de conformidad con el marco general propio de la psicología del trabajo y de la psicología de la salud en el trabajo (p.ej., Barling \& Griffiths, 2003; Benavides, Ruiz Frutos \& García, 1997; Bennett, Cook \& Pelletier, 2003; Brodsky, 1996; Cabral, 1988; Castejón, 1997; Quick \& Tetrick, 2003; Schabracq, Winnubst, \& Cooper, 2003; Smith et al., 2003).

\section{FUNCIÓN DEL MODELO}

Se trata de un modelo para explicar las consecuencias del trabajo sobre el trabajador en el día a día de la jornada laboral, (a) midiendo cómo son las demandas cuantitativas y cualitativas del trabajo que recibe (condiciones de trabajo), (b) evaluando también cuáles son los recursos con los que cuenta el trabajador para afrontar tales demandas y cómo percibe éste su entono psicosocial (condiciones de adaptación), (c) estimando cuál es el ajuste o discrepancia logrados entre trabajo y trabajador (condiciones de ajuste); y examinando, finalmente, cómo afecta todo ello al BLPS y SML del trabajador (condiciones de experiencia subjetiva). Su objetivo es doble: (a) evaluar primariamente los posibles factores de riesgo psicosocial presentes en la situación de trabajo -p.ej., cantidad y/o dificultad excesiva de trabajo, ambiente hostil hacia el trabajador, falta de recursos personales del trabajador, desajustes entre trabajo y trabajador...-; y (b) explicar los efectos de estos factores antecedentes sobre el bienestar y salud del trabajador. Por lo demás, el modelo conceptúa que la SML del trabajador está anidada en su BLPS, al igual que defienden otros autores (p.ej. Danna \& Griffin, 1999). 
Entre otras características del modelo que nos ocupa merecen destacarse (a) su carácter sistemático e integrador de diversos factores que influencian el BLPS y la SML y que habitualmente se tratan por separado, y (b) su coordinación y disposición metódica de las condiciones estructurales y moduladoras componentes.

\section{Estructura factorial de LAS ESCALAS}

El análisis factorial de las escalas de medida correspondientes a sus diversas condiciones teóricas ha revelado que los factores extraídos de las escalas explican un elevado porcentaje de varianza común (Duro, 2005b; Duro, 2007b). En concreto, la varianza media explicada para las escalas de cada condición ha sido: $69,74 \%$ para las condiciones de trabajo, $57,93 \%$ para las condiciones de adaptación, 50,04\% para las condiciones de ajuste; $67,12 \%$ para las condiciones de experiencia subjetiva; y $85,12 \%$ para las condiciones moduladoras. En particular, los factores extraídos del BLPS y SML se organizan según la dimensiones básicas del trabajador -afectiva, cognitiva, conductual, física y social-, y según sea la naturaleza de la consecuencia del trabajo -positiva o negativa.

\section{HiPótesis}

De conformidad con los presupuestos del modelo en este estudio se toman como antecedentes las condiciones de trabajo, las condiciones de adaptación, las condiciones de ajuste y las condiciones de modulación del trabajador; y se toman como consecuentes sus condiciones de experiencia subjetiva, esto es su BLPS y SML además, y dada la anidación propuesta de la salud dentro del bienestar, se toma también el BLPS como antecedente de la SML (Duro, 2005a). Consiguientemente, y por las razones expuestas, podemos concluir que las condiciones antecedentes explicarán las condiciones consecuentes.

4.1. Hipótesis 1a: Las condiciones de trabajo, las condiciones de adaptación, las condiciones de ajuste y las condiciones moduladoras explicarán el BLPS de los trabajadores (valor explicativo general del modelo).

4.2. Hipótesis $1 b$ : Todas y cada una de las diferentes escalas de medida de las condiciones de trabajo, condiciones de adaptación, condiciones de ajuste y condiciones moduladoras explicarán la escala de BLPS de los trabajadores (valor explicativo particular de las escalas del modelo).

4.3. Hipótesis 2a: Las condiciones de trabajo, las condiciones de adaptación, las condiciones de ajuste, las condiciones moduladoras y el BLPS explicarán la SML de los trabajadores (valor explicativo general del modelo).

4.4. Hipótesis $2 b$ : Todas y cada una de las diferentes escalas de medida de las condiciones de trabajo, condiciones de adaptación, condiciones de ajuste, condiciones moduladoras, así como también la escala BLPS explicarán la escala de SML de los trabajadores (valor explicativo particular de las escalas del modelo).

\section{MÉTodo}

\subsection{Muestra}

La muestra estuvo compuesta por un total de 719 trabajadores, con una edad media de 32,87 años $(\mathrm{Dt}=9,65)$. El 49,3\% de los cuales eran varones. En su clasificación profesional, destacan las siguientes categorías ocupacionales: administrativos, $39,8 \%$; técnicos, $28,5 \%$; comerciales, $12,1 \%$; y operarios, $10,6 \% 3 / 4$ sólo un $1,5 \%$ del total de la muestra ocupaban puestos directivos. En su gran mayoría, los trabajadores de la muestra procedían de las empresas y red social de aquellos de nuestros alumnos que participaron en este estudio para obtener los créditos de su trabajo práctico -también algunas pocas empresas de tamaño medio tomaron parte en este estudio. Todos los sujetos de la muestra participaron voluntariamente. Por lo demás, tanto los alumnos como los trabajadores evaluados desconocían las hipótesis de trabajo últimas de la presente investigación.

\subsection{Medidas}

En este trabajo se tomarán como medidas antecedentes (variables explicativas) los factores previamente extraídos de cada escala de medida del modelo, según detallamos a continuación. Condiciones antecedentes: (a) Condiciones de trabajo: Escala de tarea-propiedades cuantitativas ( 1 factor): carga de trabajo; Escala de tareapropiedades cualitativa ( 2 factores): complejidad 
del trabajo y definición del trabajo; Escala socialpropiedades cuantitativas ( 1 factor): cantidad de contactos sociales; y Escala social-propiedades cualitativas ( 6 factores): aceptación recibida del conjunto de role, conflictos con compañeros, conflictos con clientes, conflictos con jefes, rechazo recibido de los jefes y rechazo recibido de los compañeros. (b) Condiciones de adaptación: Escala de recursos laborales del trabajador (2 factores): recursos personales generales y recursos personales sociales; Escala de atribuciones sociales ( 3 factores): hostilidad percibida en jefes, hostilidad percibida en compañeros y hostilidad percibida en la tarea; (c) Condiciones de ajuste: Escala de ajustecantidad ( 2 factores): ajuste de la cantidad de trabajo y ajuste de la cantidad de contactos sociales; Escala de ajuste-calidad (2 factores): ajuste de la aceptación recibida del conjunto de role y ajuste de la complejidad del trabajo; y Escala de ajuste-recursos ( 2 factores): ajuste de recursos personales generales y ajuste de recursos sociales. (d) Condiciones moduladoras: Escala moduladora del trabajo ( 7 factores): modulación de la cantidad de trabajo, modulación del ritmo de trabajo, modulación del contacto con clientes, modulación del contacto con compañeros, modulación de la monotonía del trabajo, modulación de la relación con clientes y modulación de la complejidad del trabajo; Escala moduladora del trabajador (6 factores): modulación personal del desgaste, modulación médica del desgaste, acotamiento del trabajo dentro de la jornada, negociación (modulación de los conflictos), desbordamiento del trabajo fuera de la jornada y modulación social del desgaste; Escala de apoyo social (2 factores). Apoyo de familiares, y apoyo de jefes y compañeros; y Escala de apoyo profesional (1 factor): apoyo profesional.

El BLPS se medirá con los siguientes siete factores extraídos de su correspondiente escala: desgaste, consecuencias afectivas positivas, consecuencias cognitivas negativas, consecuencias conductuales negativas, consecuencias físicas negativas, consecuencias sociales negativas, y absentismo; y la SML se medirá con estos nueve factores extraídos de su escala: trastorno emocional general, trastornos físicos, miedo, alteraciones perceptivascognitivas, abuso del tabaco, ajuste perceptivocognitivo, refuerzo emocional, abuso de alcohol y drogas, y accidentes laborales.
Las características estructurales del instrumento de medida original, Encuesta de calidad de vida laboral y salud mental en el trabajo ${ }^{\circledR}$, y los resultados del análisis factorial figuran en Duro (2005b; Duro, 2007b).

\section{Resultados}

Por tratarse las medidas de puntuaciones factoriales todas sus medias son iguales a cero y sus desviaciones típicas iguales a uno. La matriz de correlaciones, dada su extensión y por razones de espacio, no se incluye en este estudio, pero el autor la remitirá a su requerimiento a las personas interesadas. En las Tablas 1 y 2 se presentan los resultados agrupados por escalas de medida de los modelos de regresión múltiple utilizados para obtener la varianza explicada (Aiken \& West, 1991; Cohen, 2003).

\subsection{RESUltados RESPECTO A HIPÓTESIS}

La hipótesis la encuentra apoyo. Los resultados indican que las condiciones de trabajo, las condiciones de adaptación, las condiciones de ajuste, y las condiciones moduladoras del explican significativamente la varianza del BLPS de los trabajadores: desgaste $\left(R^{2}=0.457 ; p<\right.$ $.001)$; consecuencias afectivas positivas $\left(R^{2}=\right.$ $0.471 ; p<.001)$; consecuencias cognitivas negativas $\left(R^{2}=0.220 ; p<.001\right)$; consecuencias conductuales negativas $\left(R^{2}=0.289 ; p<.001\right)$; consecuencias físicas negativas $\left(R^{2}=0.211 ; p<\right.$ $.001)$; consecuencias sociales negativas $\left(R^{2}=\right.$ $0.448 ; p<.001)$; y absentismo $\left(R^{2}=0.142 ; p<\right.$ $.001)$.

La Hipótesis 1 encuentra apoyo. Todas y cada una de las escalas de medida de las condiciones de trabajo, condiciones de adaptación, condiciones de ajuste, y condiciones moduladoras explican significativamente la varianza de al menos alguno de los factores del BLPS. La escala social: propiedades cualitativas, la Escala moduladora del trabajo y la Escala moduladora del trabajador explican significativamente la varianza de todos y cada uno de los siete factores del BLPS; en tanto que la Escala de tarea: propiedades cuantitativas explica significativamente la varianza de sólo tres factores del BLPS. Las restantes escalas explican cada una significativamente la varianza de entre cuatro y seis factores del BLPS (ver Tabla 1). 
La hipótesis 2 a encuentra un apoyo parcial (aunque mayoritario). Las condiciones de trabajo, las condiciones de adaptación, las condiciones de ajuste, el BLPS, y las condiciones moduladoras explican significativamente la varianza de la SML: trastorno emocional general $\left(R^{2}=0.557 ; p<.001\right)$, trastornos físicos $\left(R^{2}=\right.$ $0.273 ; p<.001)$, miedo $\left(R^{2}=0.293 ; p<.001\right)$, alteraciones perceptivo-cognitivas $\left(\left(R^{2}=0.557\right.\right.$; $\left.p<.001), R^{2}=0.208 ; p<.001\right)$, ajuste perceptivo-cognitivo $\left(R^{2}=0.311 ; p<.001\right)$, refuerzo emocional $\left(R^{2}=0.386 ; p<.001\right)$, abuso del alcohol y drogas $\left(R^{2}=0.267 ; p<.001\right)$, y accidentes laborales $\left(R^{2}=0.135 ; p<.01\right)$. Por el contrario, no se explica significativamente la varianza del abuso del tabaco $\left(R^{2}=0.072\right.$; n.s. $)$.

La hipótesis $2 b$ encuentra apoyo. Todas y cada una de las escalas de medida correspondientes a las condiciones de trabajo, condiciones de adaptación, condiciones de ajuste, condiciones moduladoras, así como la escala del BLPS explican significativamente la varianza de al menos alguno de los factores de la SML de los trabajadores. El valor explicativo máximo lo ostentan la Escala social: propiedades cualitativas y la Escala de BLPS que explican significativamente la varianza de todos y cada uno de los factores de la SML -a excepción del factor de abuso del tabaco; correspondiendo el menor valor explicativo a la Escala social: propiedades cuantitativas que explica significativamente la varianza de sólo dos factores de la SML del trabajador. El resto de las escalas explican cada una entre siete y tres factores del SML (ver Tabla 2).

\section{Discusión}

Nuestros resultados indican que las condiciones de trabajo, condiciones de adaptación, condiciones de ajuste, y condiciones moduladoras explican significativamente el BLPS de los trabajadores, según postula el modelo conjunto de calidad de vida y salud mental en el trabajo. El valor explicativo de este modelo es elevado en el caso del desgaste del trabajador, y también en el caso de las consecuencias afectivas positivas y consecuencias sociales negativas que experimenta el trabajador por causa de su trabajo. Por el contrario, la validez explicativa del absentismo resulta pequeña -aunque significativa. Para el resto de los componentes del BLPS del trabajador-consecuencias cognitivas negativas, consecuencias conductuales negativas y consecuencias físicas negativas- el valor explicativo es significativo y de tipo medio. Destaca en particular la amplitud de la validez explicativa que muestran las tres escalas siguientes: Escala social: propiedades cualitativas, Escala moduladora del trabajo y Escala moduladora del trabajador -lo que pone de manifiesto, una vez más, la enorme importancia que reviste para el BLPS del trabajador el tono y clima de sus relaciones sociales en el puesto de trabajo (Fiske \& Yamamoto, 2005; Frone, 2000), y el papel principal que juegan las estrategias moduladoras desplegadas por el trabajador.

Asimismo, nuestros resultados indican que las condiciones de trabajo, las condiciones de adaptación, las condiciones de ajuste, las condiciones moduladoras, y el propio BLPS explican significativamente la SML del trabajador, según se contempla en el modelo conjunto de calidad de vida y salud mental en el trabajo. La varianza explicada del trastorno emocional general del trabajador y del refuerzo emocional que recibe para amortiguar los daños sobre su salud resulta especialmente elevada únicamente el modelo no consigue explicar el abuso del tabaco por causa del trabajo. Sobresale, en primer lugar, el alcance de la validez explicativa de la Escala social: propiedades cualitativas y también de la Escala de BLPS resultado este último que refuerza la idea de que la SML del trabajador está anidada en su BLPS. En segundo lugar, se distingue el valor explicativo de la Escala moduladora del trabajador y de las escalas de Atribuciones sociales y de Ajuste: cantidad, que también demuestran una amplitud explicativa considerable -lo que indica el papel trascendental que juega la dimensión social del trabajo y la estrategia auto-moduladora del trabajador en la salud mental laboral, sin olvidar la carga material del trabajo que se debe realizar.

Como se aprecia en nuestros resultados, todas las escalas del modelo contribuyen a explicar la varianza, ayudando y concurriendo unas con otras para explicar conjuntamente el BLPS y SML de los trabajadores, tal y como cabría esperar en un modelo de carácter integrado.

En comparación con otros modelos y cuestionarios relacionados, la varianza aquí explicada es superior a la que consiguen explicar el JCQ, Job Content Questionnaire (Hastings et 
al, 2004; Karasek et al, 1998); el MBI, Maslach Burnout Inventory (Demerouti et al, 2003; Maslach \& Jackson, 1986); el RSI, Reeder Stress Inventory (Reeder, Chapman \& Coulson, 1968); y las escalas de mecanismos de afrontamiento y estrés de Frese (1986) - por citar tan sólo algunos ejemplos.

A tenor de estos resultados, estimamos que este modelo puede resultar útil para la gestión de RR.HH. en las empresas y organizaciones de cara a una evaluación primaria de los riesgos psicosociales de su plantilla-función evaluadora del modelo-, como paso previo y necesario para adoptar medidas de prevención (Duro, 2006; Meliá et al, 2006) e intervención al nivel que sea conveniente (Duro, 2007a). Una evaluación preventiva regular y sistemática de del bienestar y salud laborales de la plantilla servirá para detectar los problemas a tiempo, y servirá para orientar sobre las adecuadas acciones correctoras que habrán de mejorar aquellas dimensiones del trabajo y/o del trabajador que vengan resultando deficientes y/o insuficientes -incluyéndola, por ejemplo, dentro del marco de los programas de ayuda al empleado (Csiernik, 2005). En definitiva, repárese en ello, una evaluación primaria del bienestar y salud de los trabajadores supondrá un ahorro de futuros costos, dada la estrecha conexión entre falta de bienestar y daños sobre la salud (Duro, 2008). Independientemente de lo anterior, desde una perspectiva más amplia, el modelo puede coadyuvar a la elaboración de una política de RR.HH. centrada en el trabajador y en la dirección de equipos (p.ej. Duro, 2006b) -función directriz del modelo-, e inclusive para calibrar el impacto que pudieran tener sobre el BLPS y la SML todos aquellos cambios organizacionales que se tenga a bien llevar a cabo en la compañía -función de seguimiento del modelo.

\subsection{Limitaciones del TRABAJO Y FUtURA INVESTIGACIÓN}

Aunque la muestra ha sido amplia, sin embargo su obtención no ha sido sistemática. Sería conveniente trabajar con una muestra adecuadamente estratificada por sectores económicos, tipos de empresa, y segmentada por categorías profesionales y variables demográficas. La evaluación de todas las condiciones del modelo se ha realizado mediante un único instrumento de medida por lo que los resultados pueden verse afectados por la varianza del método común (Spector, 1987). Finalmente, el diseño ha sido transversal. Sería deseable que la investigación futura superara estas limitaciones. 


\section{REFERENCIAS BiBLIOGRÁFICAS}

Aiken, L. S., \& West, S. G. (1991). Multiple regression: Testing and interpreting interactions. Newbury Park, CA: Sage.

Barling, J. \& Griffiths, A. (2003). A History of Occupational Health Psychology. En Quick, J.C. \& Tetrick, L.E. (Eds.). Handbook of occupational health psychology (pp. 19-33). Washington, DC: APA.

Benavides, F.C., Ruíz-Frutos, C. \& García, A.M. (1997). Salud laboral. Conceptos y técnicas para la prevención de riesgos laborales. Barcelona: Masson (cap. 3, pp. 37-48).

Bennett, J.B., Cook, R.F. \& Pelletier, K.R. ( 2003). Toward an Integrated Framework for Comprehensive Organizational Wellness: Concepts, Practices, and Research in Workplace Health Promotion. En Quick, J.C. \& Tetrick, L.E. (Eds). Handbook of occupational health psychology (pp. 69-95). Washington, DC: APA.

Brodsky, C.M. (1996). The psychiatry of work. Occupational Medicine, 11(4), 685-698.

Cabral, C.A. (1988). Psicopatología del trabajo. Acta Psiquiátrica y Psicológica de América Latina, 34 (3), 237-242.

Castejón, E. (1997). Vigilancia de los factores de riesgo. En: Benavides, F.G., Ruiz, C. \& García, A.M (Eds.). Salud laboral. Conceptos y técnicas para la prevención de riesgos laborales. Barcelona: Masson (cap. 21, pp. 215-224).

Cohen, J. (2003). Applied Multiple Regression - Correlation Analysis for the Behavioural Sciences. Mahwah, NJ: Lawrence Erlbaum Associates.

Cooper, C.L. (1998). Theories of organizational stress. New York: Oxford University Press.

Cooper, C.L. \& Marshall, J. (1978). Understanding Executive Stress. London: MacMillan.

Csiernik, R. (2005). What We Are Doing in the Employee Assistance Program: Meeting the Challenge of the Integrated Model of Practice. Journal of Workplace Behavioral Health, 21(1), 11-22.

Danna, K., \& Griffin, R.W. (1999). Health and well-being in the workplace: A review and synthesis of the literature. Journal of Management, 25 (3), 357-384.

Demerouti, E., Bakker, A.B., Vardakou, I., \& Kantas, A. (2003). The convergent Validity of Two Burnout Instruments. A Multitrait-Multimethod Analysis. European Journal of Psychological Assement, 19 (1), 12-23.

Duro, A. (2008) Poor employee well-being and wok-related mental ill-health and substance consumption: A model on the relationship between non-clinical and clinical consequences of work. Manuscrito no publicado

(2007b). Modelo de componentes comunes para explicar el bienestar laboral psicológico y la salud mental laboral del trabajador: estructura factorial de sus escalas de medida. Manuscrito no publicado.

(2007a). Factores individuales. Medidas preventivas. En: Foment del Treball Nacional (ed.). Perspectivas de Intervención en Riesgos Psicosociales, Capítulo, 4, páginas 91-112. Barcelona: Foment del Treball Nacional.

(2006b). Modelo conjunto de calidad de vida laboral y salud mental. En: Foment del Treball Nacional (ed.). Perspectivas de Intervención en Riesgos Psicosociales. Evaluación de riesgos, Capítulo 8, páginas 181-209. Barcelona: Foment del Treball Nacional.

(2006a). Introducción al Liderazgo Organizacional. Teoría y metodología. Universidad Rey Juan Carlos, Facultad de Ciencias Jurídicas y Sociales, ${ }^{\circ}$ 46. Madrid: Dykinson.

(2005a). Calidad de Vida Laboral y Psicología Social de la Salud Laboral: hacia un modelo de componentes comunes para explicar el bienestar laboral psicológico y la salud mental laboral de origen psicosocial. Fundamentos teóricos. Revista del Ministerio de Trabajo y Asuntos Sociales. Serie Economía y Sociología, 56, 15-56.

(2005b). Calidad de Vida Laboral y Psicología Social de la Salud Laboral: hacia un modelo de componentes comunes para explicar el bienestar laboral psicológico y la salud mental laboral de origen psicosocial. Resultados preliminares. Revista del Ministerio de Trabajo y Asuntos Sociales. Serie Economía y Sociología, 56, 57-98.

Fiske, S. T., \& Yamamoto, M. (2005). Coping With Rejection: Core Social Motives Across Cultures. In: D.K. Williams, J. P. Forgas, \& W. von Hippel, (Eds), The social outcast: Ostracism, social exclusion, rejection, and bullying. New York, NY: Psychology Press. 
Frese, M. (1986). Coping as a moderator and mediator between stress at work and psychosomatic complaints. En M.H. Appley \& R. Trumbull (Eds.), Dynamics of Stress. Planum Press: New York. Freudenberger, H.J. (1974). Staff burn-out. Journal of Social Issues, 30 (1), 159-165.

Frone, M. R. (2000). Interpersonal conflict at work and psychological outcomes: Testing a model among young workers. Journal of Occupational Health Psychology, 5(2), 246-255.

Harned, M.S., Ormerod, A.J., Palmieri, P.A., Collinsworth, L.L. \& Reed, M. (2002). Sexual Assault and Other Types of Sexual Harassment by Workplace Personnel: A Comparison of Antecedents and Consequences. Journal of Occupational Health Psychology, 7 (2), 174-188.

Hastings, R.P., Horne, S. \& Mitchell, G. (2004). Burnout in direct care staff in intellectual disability services: a favor analytic study of the Maslach Burnout Inventory. Journal of Intellectual Disability Research, 48 (3), 268-273.

Hodson, R., Roscigno, V.J., \& López, Seven. H. (2006). Chaos and the Abuse of Power. Workplace Bullying in Organizational and Interactional Context. Work and Occupations, 33 (4), 382-416.

Karasek, R.A. (1979). Job Demands, Job Decisión Latitude, and Mental Strain: Implications for Job Redesign (1979). Administrative Science Quarterly, 24(2), 285-307.

Karasek, R., Brisson, Ch., Kawakami, N., Houtman, I. \& Bongers, P. (1998). The Job Content Questionnaire (JCQ): An Instrument for Internationally Comparative Assessments of Psychosocial Job Characteristics. Journal of Occupational Health Psychology, 3 (4), 322-355.

Lee, R. \& Asforth, B.E. (1996). A meta-analytic examination of the correlates of the three dimensions of job burnout. Journal of Applied Psychology, 81(2), 123-133

Maslach, C. (1982). Burnout: The cost of caring. New York: Prentices-Hall Press.

Maslach, C. (1986). Stress, burnout, and workacoholism. En R.R. Kilberg, P.E. Mathan, \& R.W. Thoreson (Eds). Professional's distress: Issues, syndromes and solutions in psychology (pp. 53-75). Washington: Psychology Ass.

Maslach, C. \& Jackson, S.E. (1981). Maslach Burnout Inventory (1986, 2a ed.). Palo Alto, CA: Consulting Psychologists Press.

Meliá, J. L.; Nogareda, C.; Lahera, M.; Duro, A.; Peiró, J. M.; Salanova, M., \& Gracia, D. (2006). Principios Comunes para la Evaluación de los Riesgos Psicosociales en la Empresa. En: Foment del Treball Nacional (Ed.). Perspectivas de Intervención en Riesgos Psicosociales. Evaluación de riesgos, Capitulo 1, páginas 9-36. Barcelona: Foment del Treball Nacional.

Peiró, J.M. (1.993). Desencadenantes del estrés laboral. Madrid: Eudema.

Phillips, J.R. (1984). Faculty burnout. American Journal Nursing, 82 (12), 1525-1526.

Pines, A. \& Aronson, E. (1988). Career burnout: Causes and cures. Nueva York: The Free Press.

Piñuel \& Zabala, I. (2001). Mobbing, como sobrevivir al acoso psicológico en el trabajo. Santander: Sal Terrae.

Quick, J.C. \& Tetrick, L.E. (2003). Handbook of occupational health psychology. Washington, DC: APA

Reeder, L.G., Chapman, J.M. \& Coulson, A.H. (1968). Socioenvironmental stress, tranquilizers and cardiovascular disease. Proceeding of the Excerpta Medica International Congress Series, 182, 226-238

Schabracq, M.J. (2003). Everyday Well-Being and Stress in Work and Organisations. En: M.J., Schabracq, A.M Winnubst,. \& C.L. Cooper, (Eds). Handbook of Work and Health Psychology, Second Edition. Chichester, UK: John Wiley \& Sons.

Shirom, A. (1989). Burnout in work organizations. En C.L. Cooper \& I. Robertson (Eds.). International review of industrial and organizational psychology (pp. 26-48). New York: Wiley.

(2003). Job-Related Burnout: A Review. En J.C. Quick, \& L.E. Tetrick, (Eds). Handbook of occupational health psychology (pp. 245-264). Washington, DC: APA.

Spector, P. E. (1987). Method variance as an artifact in self-reported affect and perceptions at work: Myth or significant problem? Journal of Applied Psychology, 72, 438-443.

Smith, M.J., Karsh, Ben-Tzion, Carayon, P. \& Conway, F.T. (2003). Controlling Occupational Safety and Health Hazards. En J.C. Quick, \& L.E Tetrick. (Eds). Handbook of occupational health psychology (pp. 35-68). Washington, DC: APA.

Spielberger, C.D. \& Vagg, P.R. (1999). Job stress Survey-Professional manual. Odessa, FL: Psychological Assessment Resources. 
Spielberger, Ch.D., Vagg, P.R. \& Wasala, C.F. (2003). Occupational Stress: Job Pressures and Lack of Support. En , J.C. Quick \& L.E. Tetrick (Eds). Handbook of occupational health psychology (pp. 185200). Washington, DC: APA.

TABLA No. 1.

Varianza explicada del bienestar laboral psicológico

\begin{tabular}{|c|c|c|c|c|c|c|c|}
\hline Variables explicativas & Desgaste & $\begin{array}{l}\text { Consecuenc. } \\
\text { afectivas } \\
\text { positivas }\end{array}$ & $\begin{array}{c}\text { Consecuenc. } \\
\text { cognitivas } \\
\text { negativas }\end{array}$ & $\begin{array}{l}\text { Consecuenc. } \\
\text { conductuales } \\
\text { negativas }\end{array}$ & $\begin{array}{l}\text { Consecuenc. } \\
\text { físicas } \\
\text { negativas }\end{array}$ & $\begin{array}{l}\text { Consecuenc. } \\
\text { sociales } \\
\text { negativas }\end{array}$ & Absentismo \\
\hline \multicolumn{8}{|l|}{ Condiciones de trabajo } \\
\hline Escala de tarea: propiedades cuantitativas & $0,140^{*}$ & $0,079^{*}$ & 0,003 & 0,002 & $0,031^{*}$ & 0,000 & 0,000 \\
\hline Escala de tarea: propiedades cualitativas & $0,101^{*}$ & $0,180^{*}$ & $0,015^{\dagger}$ & $0,016^{\dagger}$ & $0,024^{*}$ & $0,022^{\dagger}$ & 0,002 \\
\hline Escala social: propiedades cuantitativas & $0,014^{\dagger}$ & $0,110^{*}$ & $0,008^{\ddagger}$ & $0,005^{\|}$ & $0,018^{\dagger}$ & $0,084^{*}$ & 0,000 \\
\hline Escala social: propiedades cualitativas & $0,207^{*}$ & $0,306^{*}$ & $0,060^{*}$ & $0,125^{*}$ & $0,072^{*}$ & $0,320^{*}$ & $0,068^{*}$ \\
\hline \multicolumn{8}{|l|}{ Condiciones de adaptación } \\
\hline Escala de recursos laborales propios & 0,004 & $0,251^{*}$ & $0,009 \|$ & $0,088^{*}$ & $0,011^{\ddagger}$ & $0,039^{*}$ & 0,002 \\
\hline Escala de atribuciones sociales & $0,102^{*}$ & 0,008 & $0,069^{*}$ & $0,041^{*}$ & $0,052^{*}$ & $0,214^{*}$ & $0,018^{\dagger}$ \\
\hline \multicolumn{8}{|l|}{ Condiciones de ajuste } \\
\hline Escala de ajuste: cantidad & $0,092^{*}$ & $0,010^{\ddagger}$ & 0,001 & 0,000 & $0,028^{*}$ & $0,017^{\dagger}$ & 0,002 \\
\hline Escala de ajuste: calidad & $0,059^{*}$ & $0,052^{*}$ & $0,047^{*}$ & $0,014^{\ddagger}$ & $0,017^{\dagger}$ & $0,096^{*}$ & 0,003 \\
\hline Escala de ajuste: recursos laborales & $0,068^{*}$ & $0,012^{\ddagger}$ & 0,003 & $0,049^{*}$ & 0,008 & $0,032^{*}$ & 0,001 \\
\hline \multicolumn{8}{|l|}{ Condiciones moduladoras } \\
\hline Escala moduladora del trabajo & $0,070^{*}$ & $0,159^{*}$ & $0,032^{\dagger}$ & $0,098^{*}$ & $0,055^{*}$ & $0,037^{\dagger}$ & $0,037^{\dagger}$ \\
\hline Escala moduladora del trabajador & $0,280^{*}$ & $0,102^{*}$ & $0,062^{*}$ & $0,023^{\ddagger}$ & $0,047^{*}$ & $0,102^{*}$ & $0,045^{*}$ \\
\hline Escala de apoyo social & $0,044^{*}$ & $0,129^{*}$ & $0,027^{*}$ & 0,006 & $0,016^{\dagger}$ & $0,102^{*}$ & 0,001 \\
\hline Escala de apoyo profesional & $0,021^{*}$ & 0,005 & $0,011^{\dagger}$ & $0,007^{\ddagger}$ & 0,002 & $0,007^{\ddagger}$ & 0,002 \\
\hline Modelo (global) & $0,457^{*}$ & $0,471^{*}$ & $0,220^{*}$ & $0,289^{*}$ & $0,211^{*}$ & $0,448^{*}$ & $0,142^{*}$ \\
\hline
\end{tabular}

Se presentan valores $R^{2}$

$* p<0.001$.

${ }^{\dagger} p<0.01$.

${ }^{\ddagger} p<0.05$.

${ }^{\mathrm{Q} \%} p<0.10$.

FUENTE: Elaboración propia 
TABLA No. 2.

Varianza explicada de la salud mental laboral

\begin{tabular}{|c|c|c|c|c|c|c|c|c|c|}
\hline Variables explicativas & $\begin{array}{c}\text { Trastorno } \\
\text { emocional } \\
\text { general }\end{array}$ & $\begin{array}{l}\text { Trastorns. } \\
\text { físicos }\end{array}$ & Miedo & $\begin{array}{l}\text { Alteracns. } \\
\text { perceptivo } \\
\text { cognitivas }\end{array}$ & $\begin{array}{l}\text { Abuso del } \\
\text { tabaco }\end{array}$ & $\begin{array}{c}\text { Ajuste } \\
\text { perceptivo } \\
\text {-cognitivo }\end{array}$ & $\begin{array}{l}\text { Refuerzo } \\
\text { emocional }\end{array}$ & $\begin{array}{c}\text { Abuso de } \\
\text { alcohol y } \\
\text { drogas }\end{array}$ & $\begin{array}{l}\text { Accidents. } \\
\text { laborales }\end{array}$ \\
\hline \multicolumn{10}{|l|}{ Condiciones de trabajo } \\
\hline Escala de tarea: prop. cuantitativas & $0,140^{*}$ & $0,012^{\dagger}$ & 0,002 & $0,018^{\dagger}$ & 0,000 & 0,118 & $0,088^{*}$ & $0,005^{\|}$ & 0,000 \\
\hline Escala de tarea: prop. cualitativas & $0,061^{*}$ & 0,008 & 0,016 & 0,002 & 0,002 & $0,080^{*}$ & $0,167^{*}$ & 0,005 & 0,002 \\
\hline Escala social: propiedades cuantitativas & 0,003 & 0,000 & 0,000 & 0,001 & 0,000 & $0,013^{\dagger}$ & $0,042^{*}$ & 0,004 & 0,003 \\
\hline Escala social: propiedades cualitativas & $0,292^{*}$ & $0,029^{\dagger}$ & $0,079^{*}$ & $0,024^{\ddagger}$ & 0,012 & $0,073^{*}$ & $0,134^{*}$ & $0,113^{*}$ & $0,031^{\dagger}$ \\
\hline \multicolumn{10}{|l|}{ Condiciones de adaptación } \\
\hline Escala de recursos laborales propios & 0,007 & 0,000 & $0,038^{*}$ & 0,003 & 0,003 & $0,041^{\star}$ & $0,069^{*}$ & $0,012^{\ddagger}$ & 0,005 \\
\hline Escala de atribuciones sociales & $0,210^{*}$ & $0,022^{\dagger}$ & $0,084^{\star}$ & $0,044^{*}$ & 0,007 & 0,002 & $0,017^{\ddagger}$ & $0,042^{*}$ & 0,008 \\
\hline \multicolumn{10}{|l|}{ Condiciones de ajuste } \\
\hline Escala de ajuste: cantidad & $0,048^{*}$ & 0,003 & $0,016^{\dagger}$ & $0,016^{\ddagger}$ & 0,006 & $0,019^{\dagger}$ & $0,043^{*}$ & $0,011^{\ddagger}$ & 0,004 \\
\hline Escala de ajuste: calidad & $0,034^{*}$ & 0,002 & $0,039^{*}$ & 0,002 & 0,001 & $0,027^{\star}$ & $0,088^{*}$ & 0,005 & 0,001 \\
\hline Escala de ajuste: recursos laborales & $0,054^{*}$ & $0,010^{l}$ & $0,074^{*}$ & 0,004 & 0,000 & 0,008 & $0,035^{*}$ & 0,006 & 0,004 \\
\hline \multicolumn{10}{|l|}{ Condicions. de experiencia subjetiva } \\
\hline Escala de bienestar laboral psicológico & $0,475^{*}$ & $0,185^{*}$ & $0,167^{*}$ & $0,086^{*}$ & 0,015 & $0,146^{*}$ & $0,320^{*}$ & $0,062^{*}$ & $0,063^{*}$ \\
\hline \multicolumn{10}{|l|}{ Condiciones moduladoras } \\
\hline Escala moduladora del trabajo & $0,076^{*}$ & 0,021 & 0,018 & $0,044^{\dagger}$ & 0,014 & $0,130^{*}$ & $0,135^{*}$ & $0,046^{*}$ & 0,006 \\
\hline Escala moduladora del trabajador & $0,240^{*}$ & $0,062^{*}$ & $0,079^{*}$ & $0,053^{*}$ & $0,018^{\|}$ & $0,054^{\star}$ & $0,064^{*}$ & $0,051^{*}$ & 0,005 \\
\hline Escala de apoyo social & $0,059^{*}$ & 0,003 & $0,012^{\ddagger}$ & $0,011^{\ddagger}$ & 0,003 & $0,021^{\dagger}$ & $0,068^{*}$ & 0,001 & 0,000 \\
\hline Escala de apoyo profesional & $0,018^{\dagger}$ & $0,060^{*}$ & $0,013^{\dagger}$ & 0,001 & 0,000 & 0,000 & 0,002 & $0,031^{*}$ & 0,001 \\
\hline Modelo (global) & $0,557^{*}$ & $0,273^{*}$ & $0,293^{*}$ & $0,208^{*}$ & 0,072 & $0,311^{*}$ & $0,386^{*}$ & $0,267^{*}$ & $0,135^{\dagger}$ \\
\hline
\end{tabular}

Se presentan valores $R^{2}$

$* p<0.001$.

${ }^{\dagger} p<0.01$.

${ }^{\ddagger} p<0.05$.

${ }^{\mathrm{Q} \%} p<0.10$.

FUENTE: Elaboración propia

FIGURA No. 1.

Modelo Teórico. Clasificación de las diferentes condiciones

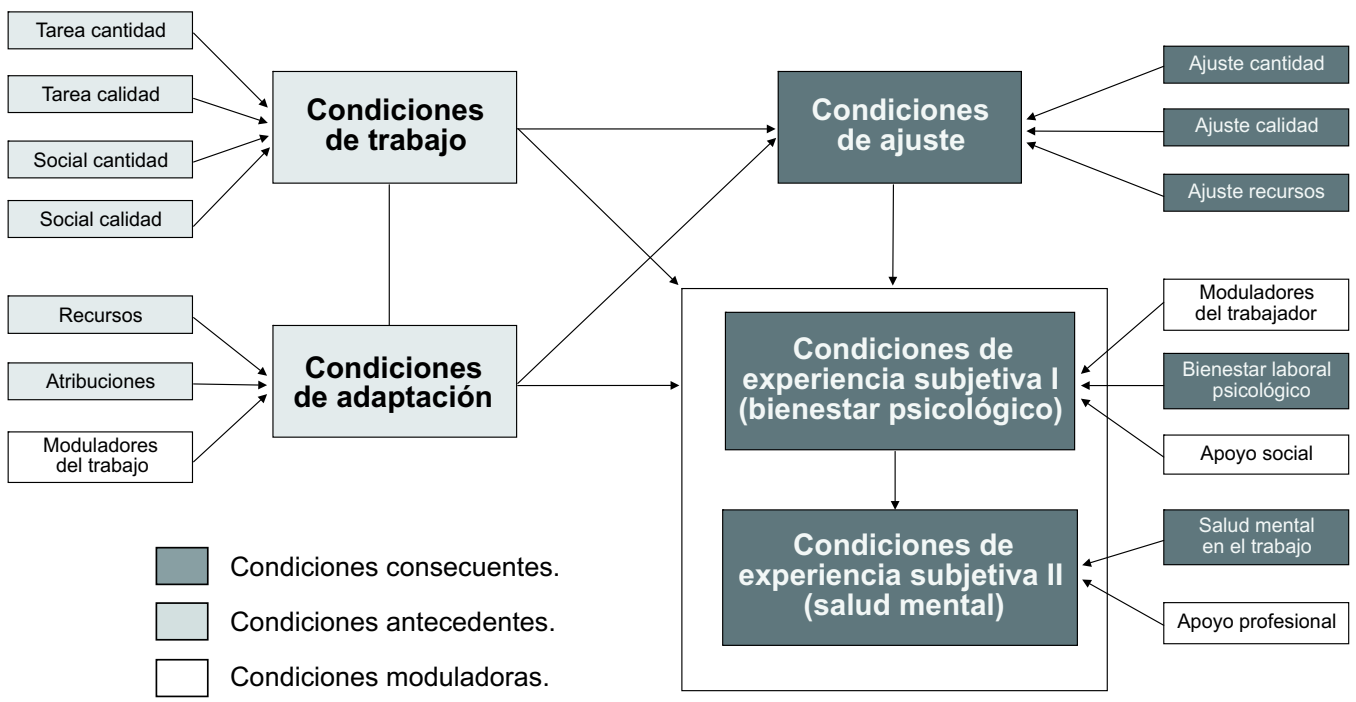

FUENTE: Elaboración propia 